\title{
Associations of sleep disturbance and duration with metabolic risk factors in obese persons with type 2 diabetes: data from the Sleep AHEAD Study
}

This article was published in the following Dove Press journal:

Nature and Science of Sleep

30 November 2012

Number of times this article has been viewed

\author{
Marie-Pierre St-Onge' \\ Gary Zammit ${ }^{2}$ \\ David M Reboussin ${ }^{3}$ \\ Samuel T Kuna ${ }^{4}$ \\ Mark H Sanders ${ }^{8}$ \\ Richard Millman ${ }^{6}$ \\ Anne B Newman ${ }^{5}$ \\ Thomas A Wadden ${ }^{4}$ \\ Rena R Wing 6 \\ F Xavier Pi-Sunyer' \\ Gary D Foster ${ }^{7}$ \\ Sleep AHEAD Research \\ Group*
}

'New York Obesity Research Center, St Luke's/Roosevelt Hospital, New York, NY, USA; ${ }^{2}$ Clinilabs, New York, NY, USA; ${ }^{3}$ Department of Biostatistical Sciences, Wake Forest School of Medicine, Winston-Salem, NC, USA; ${ }^{4}$ Department of Psychiatry, University of Pennsylvania, Philadelphia, PA, USA; ${ }^{5}$ Department of Epidemiology, University of Pittsburgh, Pittsburgh, PA, USA; ${ }^{6}$ Department of Psychiatry and Human Behavior, Brown University, Providence, RI, USA; ${ }^{7}$ Center for Obesity Research and Education,

Temple University, Philadelphia, PA, USA; ${ }^{8}$ Department of Medicine, Division of

Pulmonary, Allergy and Critical Care

Medicine, University of Pittsburgh,

PA, USA

*A full list of the members of the Sleep AHEAD Research Group is available in an online appendix

Correspondence: Marie-Pierre St-Onge New York Obesity Research Center, St Luke's/Roosevelt Hospital, 1090

Amsterdam Avenue, Suite I4D,

New York, NY 10025, USA

$\mathrm{Tel}+\mathrm{I} 2125233564$

Fax + I $212523357 \mid$

Email ms2554@columbia.edu
Purpose: Some studies have found an association between sleep disturbances and metabolic risk, but none has examined this association in individuals with type 2 diabetes. The objective of this study was to determine the relationship between sleep disturbances and metabolic risk factors in obese patients with type 2 diabetes.

Patients and methods: This study was a cross-sectional examination of the relationship between sleep parameters (apnea/hypopnea index [AHI], time spent in various sleep stages) and metabolic risk markers (fasting glucose, hemoglobin $\mathrm{A}_{1 \mathrm{c}}$, lipids) using baseline data of the Sleep AHEAD cohort. Subjects $(n=305)$ were participants in Sleep AHEAD (Action for Health in Diabetes), a four-center ancillary study of the Look AHEAD study, a 16-center clinical trial of overweight and obese participants with type 2 diabetes, designed to assess the long-term effects of an intensive lifestyle intervention on cardiovascular events. All participants underwent one night of in-home polysomnography and provided a fasting blood sample. Regression analyses estimated the relationship between sleep variables and metabolic risk factors. Models were adjusted for study center, age, sex, race/ethnicity, waist circumference, smoking, alcohol intake, diabetes duration, and relevant medications.

Results: Of 60 associations tested, only one was significant: fasting glucose was associated with sleep efficiency (estimate $-0.53 \pm$ [standard error] $0.26, P=0.041$ ). No associations were found between any of the sleep variables and lipid profile or hemoglobin $\mathrm{A}_{1 \mathrm{c}}$.

Conclusions: The present data show only weak associations between select sleep variables and metabolic risk factors and do not provide strong support for a role of sleep on metabolic abnormalities in obese patients with type 2 diabetes.

Keywords: obesity, obstructive sleep apnea, metabolic risk, sleep, diabetes

\section{Introduction}

Recent studies have suggested that sleep duration, as well as sleep disturbances, may play a role in the development of metabolic abnormalities. Chaput et $\mathrm{al}^{1}$ reported that short or long sleep duration increased the risk for incident diabetes over a 6-year follow-up period in the Quebec Family Study. Individuals with short sleep duration, defined as those sleeping $\leq 6$ hours/night, also had higher fasting plasma insulin and insulin resistance, assessed by the homeostatic model (HOMA-IR), than average sleepers, defined as those sleeping 7-8 hours/night. ${ }^{1}$ A similar association between sleep duration and incident diabetes was reported from the Insulin Resistance and Atherosclerosis Study. ${ }^{2}$ In the Sleep Heart Health Study, ${ }^{3}$ a relationship between sleep duration and impaired glucose tolerance and diabetes was also observed. Data from the Hordaland Health Study suggest a relationship between short sleep duration and 
elevated total cholesterol and triglyceride, and lower highdensity lipoprotein (HDL) cholesterol levels. ${ }^{4}$

Studies of sleep restriction in healthy adults also support a role for sleep duration in the etiology of metabolic disturbances. For example, reductions in sleep by approximately 2 hours/night increased 2-hour glucose and area under the curve for glucose during a 3-hour oral glucose tolerance test,${ }^{5}$ as well as reduced insulin sensitivity, glucose tolerance, and the disposition index, ${ }^{6}$ relative to longer sleep duration. Sleep restriction has also been shown to increase total and low-density lipoprotein (LDL) cholesterol in postmenopausal women. $^{7}$

Sleep disorders such as sleep apnea have also been associated with increased risk of incident diabetes. ${ }^{8}$ In a convenience sample of individuals with type 2 diabetes presenting at a primary care and endocrinology clinic, those with severe obstructive sleep apnea (OSA) had more diabetes complications and poorer glycemic control than individuals with less severe OSA. ${ }^{9}$ Intervention studies have also shown that suppression of slow-wave sleep for three nights reduces insulin sensitivity with reductions in glucose disposition index and glucose tolerance compared to undisrupted sleep in healthy, lean young men and women. ${ }^{10}$ Studies have also suggested that the presence of OSA is associated with higher odds of metabolic syndrome ${ }^{11}$ and higher levels of metabolic syndrome indicators (blood pressure, fasting glucose, triglycerides) than those without OSA. ${ }^{11,12}$ In the São Paulo Epidemiologic Sleep Study, severity of OSA was significantly associated with impaired fasting glucose and HOMA, such that those with moderate OSA were 1.67 times more likely to have impaired fasting glucose or type 2 diabetes, and those with moderate-to-severe OSA were twice as likely to have insulin resistance than participants without OSA. ${ }^{13}$

Epidemiological and clinical studies in healthy individuals point to a link between sleep duration and disturbances and diabetes risk. However, Björkelund et a ${ }^{14}$ found no association between sleep duration, complaints, or medications and 32-year incidence of diabetes in a population study of women, and there was no relationship between sleep duration and HOMA-IR in the Wisconsin Sleep Cohort Study. ${ }^{15}$ In clinical studies, Bosy-Westphal et a ${ }^{16}$ found no effect of sleep restriction on glucose, insulin, and insulin sensitivity in healthy women. It has been proposed that there may be a threshold of minimum sleep required to maintain normal glucose control and that clinical studies showing the greatest difference in sleep duration between test periods show an effect of sleep on glucose metabolism. ${ }^{17,18}$ It is also possible that the measurements used to assess insulin resistance were not sensitive enough to detect effects of sleep duration on this marker of diabetes risk.

The present study used data from the Sleep AHEAD (Action for Health in Diabetes) cohort to determine whether sleep duration and disturbances were associated with metabolic abnormalities in obese, type 2 diabetic individuals. Based on epidemiological findings, it was hypothesized that shorter sleep duration and higher AHI would both be associated with a more adverse metabolic profile. Exploratory analyses were performed to determine if components of the sleep architecture would be specifically related to metabolic risk factors.

\section{Methods}

\section{Participants}

Participants for this study were enrolled in the Sleep AHEAD Study, whose purpose is to examine the effects of weight loss on sleep-disordered breathing in obese patients with OSA and type 2 diabetes over a 4 -year period. Although Sleep AHEAD is a longitudinal study, the present study reports on baseline, cross-sectional data. Sleep AHEAD is a four-center, ancillary study of Look AHEAD, a 16-center clinical trial investigating the long-term health impact of an intensive lifestyle intervention in 5145 overweight or obese adults with type 2 diabetes. The details of Look AHEAD's design, ${ }^{19}$ participant characteristics at baseline, ${ }^{20}$ and intervention ${ }^{21}$ (including inclusion and exclusion criteria) have been described elsewhere. Primary inclusion criteria for Look AHEAD were age 45-75 years, body mass index (BMI) $\geq 25 \mathrm{~kg} / \mathrm{m}^{2}$ ( $\geq 27$ if currently taking insulin), physician-verified type 2 diabetes, hemoglobin $\mathrm{A}_{1 \mathrm{c}}$ $\left(\mathrm{HbA}_{1 \mathrm{c}}\right)<11 \%$, and arterial pressure $<160 \mathrm{mmHg}$ systolic and $<100 \mathrm{mmHg}$ diastolic. In addition to Look AHEAD criteria, exclusion criteria for Sleep AHEAD were surgical or medical treatment for OSA. Those with previously diagnosed but untreated OSA were eligible. In the Sleep AHEAD population, $86.6 \%$ of participants had mild to severe OSA. ${ }^{22}$

\section{Procedures}

Sleep AHEAD participants were recruited at four of the Look AHEAD sites: University of Pennsylvania (Philadelphia, PA), University of Pittsburgh (Pittsburgh, PA), St Luke's/ Roosevelt Hospital (Columbia University, New York, NY), and Brown University (Providence, RI). The study was approved by the institutional review boards at each of the participating sites, and all participants provided written informed consent. At the second Look AHEAD screening visit, a research assistant informed subjects about Sleep AHEAD. 
Interested participants then consented to a screening to assess eligibility. Each Sleep AHEAD site enrolled participants until its target levels (approximately 75 per site or 300 across sites) were achieved. Total enrollment in Sleep AHEAD was 306.

\section{Polysomnography}

Baseline polysomnograms were performed prior to any intervention from the Look AHEAD study and were done in the participants' home with a portable monitor (PS2; Compumedics, Abbotsford, Australia) using techniques similar to those developed for the Sleep Heart Health Study. ${ }^{23}$ The participants were prepared for recording in their homes by two sleep technologists. Participants were instructed to go to bed at their regular time, and the monitors were programmed to start recording 1 hour earlier. Technologists returned to the participants' homes the following morning to remove the sensors and retrieve the monitors. The initial failure rate for baseline Sleep AHEAD polysomnography (PSG) recordings was $7.2 \%$ (22 of 306). Among the 22 participants with failed studies, twelve had successful repeat testing, nine declined further testing, and one failed testing on the second attempt, resulting in an actual failure rate of 3.3\% (ten of 306), which is similar to the $5.3 \%$ failure rate found in the Sleep Heart Health Study. ${ }^{24}$

All polysomnograms were scored manually with the aid of computer software by the same registered PSG technologist. The scoring of sleep stages, apneas, and hypopneas was performed in accordance with the criteria of the American Academy of Sleep Medicine criteria, using a 4\% oxygen desaturation cutoff to score hypopneas. ${ }^{25,26}$ Intrascorer reliability was determined by having the blinded scorer rescore 32 randomly chosen Sleep AHEAD polysomnograms. The intraclass correlation coefficient for AHI was 0.89 . A value $>0.80$ is considered excellent. ${ }^{27}$ Sleep variables considered in this study included: total sleep time, percent time spent in each sleep stage, sleep efficiency ( $\%$ time asleep), arousal index (average number of arousals per hour of sleep), average desaturation/apnea-hypopnea (percent change in $\mathrm{Hb}$ oxygen saturation), number of wake episodes, and AHI.

The following data (dependent variables) were obtained from the Look AHEAD study files: $\mathrm{HbA}_{1 \mathrm{C}}$, glucose, triglycerides, total cholesterol, LDL-cholesterol, and HDLcholesterol. These tests were obtained at the time that subjects entered the Look AHEAD study. All metabolic variables were measured from fasting blood samples. ${ }^{19}$

For the statistical analysis, an indicator variable was used for whether or not the participant was taking a diabetes or lipid-lowering medication. Six dependent variables and ten sleep parameters were analyzed. For each dependent variable, ten linear regression models were fit, one for each sleep parameter separately. Each model was adjusted for study site, age, sex, race/ethnicity, waist circumference, smoking (coded as never, past, present), alcohol, and diabetes duration. In addition, $\mathrm{HbA}_{1 \mathrm{c}}$ and fasting glucose were adjusted for use of diabetes medication, and lipid variables (total, LDL-, and HDL-cholesterol, and triglycerides) were adjusted for use of lipid-lowering medication. Medication use was assessed at home visits. The residuals and measures of collinearity were also checked for all 60 models; there was no indication that modeling assumptions were violated. Results are reported as estimated slope coefficients \pm standard error for each regression. Results were not adjusted for multiple comparisons. A total of 305 participants were included in the analysis; one participant with central sleep apnea ( $>50 \%$ of apneas as central apneas) was excluded.

\section{Results}

On average, participants had a BMI of approximately $36.5 \pm 0.3 \mathrm{~kg} / \mathrm{m}^{2}$ and an age of $61 \pm 0.4$ years. Study participants slept on average $5.96 \pm 1.21$ hours/night and had an AHI of $29.8 \pm 19.4$ events/hour. Diabetes duration was $7.3 \pm 0.4$ years. Except for HDL-cholesterol, baseline metabolic variables were elevated (Table 1).

There was no significant independent association between any of the sleep variables and $\mathrm{HbA}_{1 \mathrm{c}}$, total cholesterol, LDL-cholesterol, or HDL-cholesterol (Table 2). There was a significant inverse association between fasting plasma glucose and sleep efficiency $(-0.53 \pm 0.26$, $P=0.041)$.

Table I Participant characteristics

\begin{tabular}{|c|c|}
\hline Characteristics & Mean (SE) \\
\hline Sex, male/female & $122 / 183$ \\
\hline Race, $\mathrm{AA} / \mathrm{C} / \mathrm{H} / \mathrm{O}$ & $58 / 223 / 10 / 14$ \\
\hline Age, years & $61.3(0.4)$ \\
\hline Waist circumference, $\mathrm{cm}$ & II $5.0(0.7)$ \\
\hline Body mass index, $\mathrm{kg} / \mathrm{m}^{2}$ & $36.5(0.3)$ \\
\hline Use of diabetes medication, $\mathrm{Y} / \mathrm{N}^{\mathrm{a}}$ & $264 / 40$ \\
\hline Use of lipid-lowering medication, $\mathrm{Y} / \mathrm{N}^{\mathrm{a}}$ & $161 / 143$ \\
\hline Hemoglobin $A_{l c}, \%$ & $7.22(0.06)$ \\
\hline Fasting plasma glucose, $\mathrm{mg} / \mathrm{dL}$ & $153.6(2.6)$ \\
\hline Total cholesterol, mg/dL & $192.4(2.2)$ \\
\hline LDL-cholesterol, mg/dL & $114.6(1.8)$ \\
\hline HDL-cholesterol, mg/dL & $45.4(0.7)$ \\
\hline Triglycerides, mg/dL & I63.6 (5.5) \\
\hline
\end{tabular}

Note: aData missing for one person.

Abbreviations: SE, standard error; AA, African-American; C, Caucasian; H, Hispanic; O, other; LDL, low-density lipid; HDL, high-density lipid. 
Table 2 Association of metabolic variables and sleep parameters

\begin{tabular}{|c|c|c|c|c|}
\hline Dependent variables & Sleep parameter & Estimate & Standard error & $P$-value \\
\hline \multirow[t]{10}{*}{ Hemoglobin $A_{I c}{ }^{a}$} & Sleep, hours & 0.053 & 0.055 & 0.340 \\
\hline & Sleep efficiency, \% time in bed as sleeping & -0.0039 & 0.0060 & 0.512 \\
\hline & $\%$ time in stage I & 0.31 & 0.42 & 0.457 \\
\hline & $\%$ time in stage 2 & -0.22 & 0.43 & 0.607 \\
\hline & $\%$ time in delta & -0.94 & 1.28 & 0.464 \\
\hline & $\%$ time in REM & -0.040 & 0.83 & 0.961 \\
\hline & Arousal index & 0.0048 & 0.0048 & 0.325 \\
\hline & Average desaturation/apnea-hypopnea & -0.052 & 0.15 & 0.731 \\
\hline & Number of wake episodes & 0.0055 & $0.007 \mid$ & $0.44 I$ \\
\hline & Apnea/hypopnea index & -0.0017 & 0.0035 & 0.625 \\
\hline Fasting plasma & Sleep, hours & -0.92 & 2.43 & 0.706 \\
\hline \multirow[t]{9}{*}{ Glucose, $\mathrm{mg} / \mathrm{dL}^{\mathrm{a}}$} & Sleep efficiency, \% time in bed as sleeping & -0.53 & 0.26 & 0.041 \\
\hline & $\%$ time in stage I & 15.76 & 17.96 & 0.381 \\
\hline & $\%$ time in stage 2 & -11.70 & 18.70 & 0.532 \\
\hline & $\%$ time in delta & 11.16 & 55.32 & 0.840 \\
\hline & \% time in REM & -25.56 & 35.70 & 0.474 \\
\hline & Arousal index & 0.13 & 0.21 & 0.520 \\
\hline & Average desaturation/apnea-hypopnea & 3.96 & 6.50 & 0.543 \\
\hline & Number of wake episodes & 0.25 & 0.31 & 0.420 \\
\hline & Apnea/hypopnea index & -0.15 & 0.15 & 0.326 \\
\hline \multirow[t]{10}{*}{ Total cholesterol, $\mathrm{mg} / \mathrm{dL}^{\mathrm{b}}$} & Sleep, hours & 1.43 & 1.90 & 0.451 \\
\hline & Sleep efficiency, \% time in bed as sleeping & -0.030 & 0.20 & 0.882 \\
\hline & $\%$ time in stage I & -2.03 & 14.04 & 0.885 \\
\hline & $\%$ time in stage 2 & 12.13 & 14.62 & 0.407 \\
\hline & $\%$ time in delta & -23.57 & 43.37 & 0.587 \\
\hline & \% time in REM & -26.07 & 27.92 & 0.351 \\
\hline & Arousal index & -0.098 & 0.16 & 0.551 \\
\hline & Average desaturation/apnea-hypopnea & -8.53 & 5.07 & 0.093 \\
\hline & Number of wake episodes & -0.040 & 0.24 & 0.867 \\
\hline & Apnea/hypopnea index & 0.23 & 0.12 & 0.050 \\
\hline \multirow[t]{10}{*}{ LDL-cholesterol, mg/dL ${ }^{\mathrm{b}}$} & Sleep, hours & 0.25 & 1.51 & 0.869 \\
\hline & Sleep efficiency, \% time in bed as sleeping & -0.076 & 0.16 & 0.636 \\
\hline & $\%$ time in stage I & -3.92 & 11.11 & 0.724 \\
\hline & $\%$ time in stage 2 & 7.85 & 11.57 & 0.498 \\
\hline & $\%$ time in delta & 9.40 & 34.34 & 0.784 \\
\hline & $\%$ time in REM & -16.73 & 22.10 & 0.450 \\
\hline & Arousal index & -0.11 & 0.13 & 0.400 \\
\hline & Average desaturation/apnea-hypopnea & -4.78 & 4.02 & 0.236 \\
\hline & Number of wake episodes & -0.093 & 0.19 & 0.625 \\
\hline & Apnea/hypopnea index & 0.10 & 0.093 & 0.260 \\
\hline \multirow[t]{10}{*}{ HDL-cholesterol, mg/dL ${ }^{\mathrm{b}}$} & Sleep, hours & 1.09 & 0.60 & 0.070 \\
\hline & Sleep efficiency, \% time in bed as sleeping & 0.065 & 0.064 & 0.311 \\
\hline & $\%$ time in stage I & -5.80 & 4.41 & 0.190 \\
\hline & $\%$ time in stage 2 & 6.94 & 4.60 & 0.132 \\
\hline & $\%$ time in delta & -1.81 & 13.70 & 0.895 \\
\hline & $\%$ time in REM & -1.63 & 8.83 & 0.853 \\
\hline & Arousal index & -0.044 & 0.052 & 0.395 \\
\hline & Average desaturation/apnea-hypopnea & -1.99 & 1.60 & 0.216 \\
\hline & Number of wake episodes & 0.016 & 0.076 & 0.837 \\
\hline & Apnea/hypopnea index & 0.00060 & 0.037 & 0.987 \\
\hline \multirow[t]{5}{*}{ Log triglycerides ${ }^{b}$} & Sleep, hours & 0.0039 & 0.027 & 0.882 \\
\hline & Sleep efficiency, \% time in bed as sleeping & -0.0017 & 0.0028 & 0.539 \\
\hline & $\%$ time in stage I & 0.24 & 0.20 & 0.215 \\
\hline & $\%$ time in stage 2 & -0.050 & 0.20 & 0.806 \\
\hline & $\%$ time in delta & -1.03 & 0.60 & 0.088 \\
\hline
\end{tabular}


Table 2 (Continued)

\begin{tabular}{lllll}
\hline Dependent variables & Sleep parameter & Estimate & Standard error & P-value \\
\hline & \% time in REM & -0.35 & 0.39 & 0.372 \\
& Arousal index & 0.0012 & 0.0023 & 0.614 \\
& Average desaturation/apnea-hypopnea & -0.049 & 0.071 & 0.489 \\
& Number of wake episodes & 0.0025 & 0.0033 & 0.451 \\
& Apnea/hypopnea index & 0.0026 & 0.0016 & 0.111 \\
\hline
\end{tabular}

Notes: Each model was adjusted for site, age, sex, race/ethnicity, waist circumference, smoking, alcohol, and diabetes duration, plus an indicator for glucose- ${ }^{\mathrm{a}}$ or lipidlowering ${ }^{\mathrm{b}}$ medication use (assessed at home visits), as appropriate.

Abbreviations: REM, rapid eye movement; LDL, low-density lipid; HDL, high-density lipid.

\section{Discussion}

This is one of few studies to examine the relationship between sleep architecture, assessed using PSG, and metabolic risk factors, specifically in obese patients with type 2 diabetes. It was hypothesized that shorter sleep duration, components of sleep architecture, and higher AHI would be associated with a more adverse metabolic profile. Although there was a significant association between sleep efficiency and fasting plasma glucose in the present study, the data do not support a relationship between sleep or AHI and metabolic risk in obese patients with type 2 diabetes. The weak association that was observed was one of many different associations tested (60 tests of associations) and may have occurred due to type 2 error. Moreover, as our analyses were exploratory, we did not adjust for multiple comparisons, and type 1 errors could be larger than stated. Additional studies are needed to provide more definitive conclusions concerning the relationship between sleep and metabolic risk in diabetics.

Study participants were obese and had diabetes. Previous studies examining the association between self-reported sleep duration and metabolic risk factors have found that short sleep increases the risk of incident diabetes. ${ }^{1,2}$ However, these studies included a wide range of participants with and without diabetes or insulin resistance at baseline and did not assess sleep-disordered breathing. It is possible that sleep characteristics play a role in the development of diabetes and cardiovascular disease, but are not important predictors of the severity of other metabolic risk factors once diabetes is established. Moreover, study participants had a small range of $\mathrm{HbA}_{1 \mathrm{c}}$ values $(7.22 \% \pm 1.07 \%)$. In a previous study with participants with a wider range of $\mathrm{HbA}_{1 \mathrm{c}}(7.7 \% \pm 1.8 \%)$, $\mathrm{HbA}_{1 \mathrm{c}}$ was significantly associated with severity of OSA in diabetic patients. ${ }^{9}$

Previous studies have found that patients with OSA have higher glucose and triglycerides, ${ }^{15,28}$ and Drager et al ${ }^{12}$ also found higher total cholesterol and LDL-cholesterol in OSA patients compared to patients without OSA. The present data did not show an association between AHI and any of the metabolic risk factors measured in this study. Of note is that participants of this study all had type 2 diabetes, whereas none of the participants in the study by Drager et al, ${ }^{12} 24 \%$ of the OSA patients, and $11 \%$ of patients without OSA had type 2 diabetes in the study by Gasa et al. ${ }^{11}$ Sharma et al ${ }^{28}$ also found that metabolic risk factors were not different between obese individuals with OSA and obese controls without OSA, both groups being free of diabetes. Furthermore, in the Sleep Heart Health Study, while AHI was associated with prevalent cardiovascular disease, there was no relationship between AHI and total cholesterol and an inverse relationship with HDL cholesterol. ${ }^{29}$ These study differences would further support our hypothesis that sleep characteristics may play a role in the determination of metabolic risk prior to the development or diagnosis of diabetes, and that diabetes masks the effects of perturbed sleep and sleep disorders on cardiovascular risk factors. However, the cross-sectional nature of the present analyses precludes definitive statements.

Some studies have also reported that poor glycemic control, assessed by $\mathrm{HbA}_{1 \mathrm{c}}$, was associated with low sleep efficiency ${ }^{30}$ and poor sleep quality, ${ }^{30,31}$ as reported by questionnaire. Actigraphy-measured sleep fragmentation and insomnia, but not sleep duration, was associated with higher fasting glucose, insulin, and HOMA index in patients with type 2 diabetes. ${ }^{32}$ Similar associations were not observed in those without type 2 diabetes. These studies suggest that poor sleep quality may be related to glycemic control; however, participants were either newly diagnosed with type 2 diabetes ( $<1$ year), ${ }^{30}$ were at low risk of sleep-related breathing disorders, ${ }^{31}$ or were young adults (approximately 45 years of age). ${ }^{32}$ These characteristics contrast with the Sleep AHEAD study population, which had longer duration of diabetes, was older, and had a high prevalence of OSA. In addition, sleep variables in the present study were directly measured using PSG.

This study tested the hypotheses that sleep characteristics, including sleep disturbances, would be related to metabolic risk profile in patients with type 2 diabetes. If sleep 
disturbances were related to metabolic profile, than it would be expected that treatment of such disturbances would result in an improvement in metabolic risk factors. However, Sharma et $\mathrm{al}^{33}$ found that continuous positive airway pressure (CPAP) for 3 months improved lipid profile and body composition, with no effect on fasting plasma glucose and insulin in a group of young obese individuals with OSA. These effects were not observed when participants underwent sham CPAP. Of note, however, is that participants had better metabolic risk profiles at the start of sham CPAP than true CPAP, and data were not adjusted for phase order or changes in body composition (although the authors note no phase order effect). Because adipose tissue distribution is strongly associated with metabolic risk factors, it is unknown whether the metabolic improvements with CPAP treatment were due to improvements in OSA or improvements in body composition. On the other hand, withdrawal of CPAP treatment for 2 weeks in individuals who had been undergoing at least 12 months of CPAP treatment did not worsen cholesterol, glucose, or insulin levels and improved triglycerides. ${ }^{34}$ These results were observed despite a return to OSA within one night of CPAP withdrawal. However, overall, study participants did not have abnormal lipid profiles or glucose metabolism at baseline. It is well known that the effects of CPAP treatment on lipid variables have provided variable results, and readers can refer to a review by Michailidis et $\mathrm{al}^{35}$ for more information on this topic. Such results support the findings of the present study, whereby OSA was not related to any of the metabolic risk factors.

The lack of association between sleep characteristics and lipid variables has been observed by others. In the Nurses' Health Study, sleep duration was not associated with lipid profile in women with type 2 diabetes. ${ }^{36}$ In the Sleep Heart Health Study, ${ }^{37}$ total cholesterol did not vary with AHI, although HDL-cholesterol was inversely associated and triglycerides positively associated with AHI in participants $<65$ years of age. No association was observed in participants older than 65 years. It is important to note that in the Sleep Heart Health Study, there was a low prevalence of type 2 diabetes, and a small proportion of participants - 16\% - had cardiovascular disease at baseline. ${ }^{29}$ Findings of the SWAN Sleep Study lend some support to the findings of this paper of an association between sleep efficiency and fasting plasma glucose. In the SWAN study, sleep efficiency was inversely related to metabolic syndrome after adjusting for race, menopausal status, health complaints, medication use, smoking, alcohol use, exercise, and BMI. ${ }^{38}$
The present data are limited by the relative homogeneity of the study population and a relatively small sample size. All participants were overweight or obese, had type 2 diabetes, and $87 \%$ had OSA ( $23 \%$ with severe OSA). ${ }^{22}$ The sample size may have been too small to detect statistically significant associations for some parameters (eg, total cholesterol and AHI, average desaturation/apnea-hypopnea; HDL-cholesterol and sleep duration; triglycerides and percent time in delta sleep) and differences between men and women could not be assessed. Also, independent associations between sleep and metabolic risk factors could have been masked by the stronger effect of obesity ${ }^{39}$ and also possibly type 2 diabetes. Age may further limit our ability to detect effects of sleep on metabolic risk factors, although age was not related to metabolic risk in the SWAN Sleep Study. ${ }^{38}$

Sleep data were obtained from a single night of in-home monitoring. Although in-home PSG is well accepted by the sleep community, ${ }^{23}$ conditions cannot be controlled. However, participants were tested in their own environment and instructed to go to bed at their usual time, but not given any instruction about specific wake-up time. This in-home testing procedure may have alleviated some of the issues surrounding a single night of PSG measurement, especially as those may arise from sleeping in an unfamiliar environment. Test-retest variability has been found to be lower with in-home PSG compared to in-lab PSG in untreated OSA patients. ${ }^{40}$ Also, although it can be argued that the data used in the present analyses may have been limited by the high night-to-night variability of $\mathrm{AHI},{ }^{40}$ there was no association between other, less variable, sleep measures and metabolic risk factors. However, Quan et $\mathrm{al}^{41}$ have previously demonstrated no consistent bias in repeated in-home PSG measurements in measures of sleep architecture and quality.

Despite its limitations, the Sleep AHEAD study provided a well-characterized population on whom to study the relationship between sleep parameters and metabolic risk. As a result, it can be concluded that within an overweight and obese group of older patients with established and generally controlled type 2 diabetes, neither sleep disturbances nor duration, assessed by PSG, are strongly associated with metabolic disorders. Sleep characteristics may be more important in individuals without established diabetes or nonobese patients. However, the Sleep AHEAD study is a longitudinal study testing the effects of weight loss on sleep-disordered breathing. Future information from this study may provide additional information concerning the relationship between sleep-disordered breathing and changes in metabolic risk factors over time. 


\section{Acknowledgments}

Sleep AHEAD was supported by the National Institutes of Health (NIH) National Heart, Lung, and Blood Institute Grant HL070301. Look AHEAD was supported by the NIH National Institute of Diabetes and Digestive and Kidney Diseases grants DK57135, DK56992, DK57002, and DK57178. This study was supported in part by HL091352. Clinical trials registration number NCT00194259, http:// www.clinicaltrials.gov. Mark H Sanders is now retired.

\section{Disclosure}

Dr Sanders is a scientific consultant to Philips-Respironics. Consistent with this he is a coinventor of the BiPAP ${ }^{\circledR}$ brand and related technologies, patents which have been assigned to Philips-Respironics for use with their BiPAP ${ }^{\circledR}$ brand and related technologies and in exchange for a royalty interest. $\mathrm{He}$ is an editor-in-chief and section editor (Sleep Medicine section) for the UpToDate website and a field editor for Sleep Medicine, and receives remuneration for these activities. He is a deputy editor for Sleep. Gary Zammit has received grant funding from Abbott, Actelion, Ancile, Apnex, Arena, Aventis, Cephalon Inc, CHDI, Elan, Epix, Evotec, Forest, Galderma, GlaxoSmithKline, H Lundbeck A/S, King, Merck and Co, Neurim, Neurocrine Biosciences, Neurogen, Organon, Orphan Medical, Otsuka, Pfizer, Predix, Respironics, Sanofi-Aventis, Sanofi-Synthelabo, Schering-Plough, Sepracor, Shire, Somaxon, Takeda Pharmaceuticals North America, Targacept, Thymon, Transcept, UCB Pharma, Predix, Vanda, and Wyeth-Ayerst Research. He is a consultant for Actelion, Alexza, Arena, Aventis, Biovail, BoehringerIngelheim, Cephalon Inc, Elan, Eli Lilly, Evotec, Forest, GlaxoSmithKline, Jazz, King Pharmaceuticals, Ligand, McNeil, Merck and Co, Neurocrine Biosciences, Organon, Pfizer, Renovis, Sanofi-Aventis, Select Comfort, Sepracor, Shire, Somnus, Takeda Pharmaceuticals, Vela, and Wyeth. Dr Zammit has also received honoraria from Neurocrine Biosciences, King Pharmaceuticals, McNeil, Sanofi-Aventis, Sanofi-Synthelabo, Sepracor, Takeda Pharmaceuticals, Vela Pharmaceuticals, and Wyeth-Ayerst Research. He is owner/ director of Clinilabs Inc, Clinilabs IPA Inc, and Clinilabs Physician Services, PC. Dr Kuna has received grant support from Philips Respironics. All other authors have no conflict of interest to disclose.

\section{References}

1. Chaput JP, Després JP, Bouchard C, Astrup A, Tremblay A. Sleep duration as a risk factor for the development of type 2 diabetes or impaired glucose tolerance: analyses of the Quebec Family Study. Sleep Med 2009;10(8):919-924.
2. Beihl DA, Liese AD, Haffner SM. Sleep duration as a risk factor for incident type 2 diabetes in a multiethnic cohort. Ann Epidemiol. 2009;19(5):351-357.

3. Gottlieb DJ, Punjabi NM, Newman AB, et al. Association of sleep time with diabetes mellitus and impaired glucose tolerance. Arch Intern Med. 2005;165(8):863-867.

4. Bjorvatn B, Sagen IM, Øyane N, et al. The association between sleep duration, body mass index and metabolic measures in the Hordaland Health Study. J Sleep Res. 2007;16(1):66-76.

5. Nedeltcheva AV, Kessler L, Imperial J, Penev PD. Exposure to recurrent sleep restriction in the setting of high caloric intake and physical inactivity results in increased insulin resistance and reduced glucose tolerance. J Clin Endocrinol Metab. 2009;94(9):3242-3250.

6. Buxton OM, Pavlova M, Reid EW, Wang W, Simonson DC, Adler GK. Sleep restriction for 1 week reduces insulin sensitivity in healthy men. Diabetes. 2010;59(9):2126-2133.

7. Kerkhofs M, Boudjeltia KZ, Stenuit P, Brohee D, Cauchie P, Vanhaeverbeek M. Sleep restriction increases blood neutrophils, total cholesterol and low density lipoprotein cholesterol in postmenopausal women: a preliminary study. Maturitas. 2007;56(2):212-215.

8. Botros N, Concato J, Mohsenin V, Selim B, Doctor K, Yaggi HK. Obstructive sleep apnea as a risk factor for type 2 diabetes. Am J Med. 2009;122(12):1122-1127.

9. Aronsohn RS, Whitmore H, Van Cauter E, Tasali E. Impact of untreated obstructive sleep apnea on glucose control in type 2 diabetes. Am $J$ Respir Crit Care Med. 2009;181(5):507-513.

10. Tasali E, Leproult R, Ehrmann DA, Van Cauter E. Slow-wave sleep and the risk of type 2 diabetes in humans. Proc Natl Acad Sci U S A. 2008;105(3):1044-1049.

11. Gasa M, Salord N, Fortuna AM, et al. Obstructive sleep apnoea and metabolic impairment in severe obesity. Eur Respir J. 2011;38(5): 1089-1097.

12. Drager LF, Lopes HF, Maki-Nunes C, et al. The impact of obstructive sleep apnea on metabolic and inflammatory markers in consecutive patients with metabolic syndrome. PLoS One. 2010;5(8):e12065.

13. Togeiro SM, Carneiro G, Ribeiro Filho FF, et al. Consequences of obstructive sleep apnea on metabolic profile: a population-based survey. Obesity (Silver Spring). Epub June 14, 2012.

14. Björkelund C, Bondyr-Carlsson D, Lapidus L, et al. Sleep disturbances in midlife unrelated to 32-year diabetes incidence: the prospective population study of women in Gothenburg. Diabetes Care. 2005;28(11): 2739-2744.

15. Taheri S, Austin D, Lin L, Nieto FJ, Young T, Mignot E. Correlates of serum C-reactive protein (CRP) - no association with sleep duration or sleep disordered breathing. Sleep. 2007;30(8):991-996.

16. Bosy-Westphal A, Hinrichs S, Jauch-Chara K, et al. Influence of partial sleep deprivation on energy balance and insulin sensitivity in healthy women. Obes Facts. 2008;1(5):266-273.

17. Donga E, van Dijk M, van Dijk JG, et al. A single night of partial sleep deprivation induces insulin resistance in multiple metabolic pathways in healthy subjects. J Clin Endocrinol Metab. 2010;95(6): 2963-2968.

18. Spiegel K, Leproult R, L'Hermite-Baleriaux M, Copinschi G, Penev PD, Van Cauter E. Leptin levels are dependent on sleep duration: relationships with sympathovagal balance, carbohydrate regulation, cortisol, and thyrotropin. J Clin Endocrinol Metab. 2004;89(11): $5762-5771$.

19. Ryan DH, Espeland MA, Foster GD, et al. Look AHEAD (Action for Health in Diabetes): design and methods for a clinical trial of weight loss for the prevention of cardiovascular disease in type 2 diabetes. Control Clin Trials. 2003;24(5):610-628.

20. Look Ahead Research Group, Bray G, Gregg E, et al. Baseline characteristics of the randomised cohort from the Look AHEAD (action for health in diabetes) study. Diab Vasc Dis Re. 2006;3(3):218-225.

21. Wadden TA, West DS, Delahanty L, et al. The Look AHEAD study: a description of the lifestyle intervention and the evidence supporting it. Obesity (Silver Spring). 2006;14(5):737-752. 
22. Foster GD, Sanders MH, Millman R, et al. Obstructive sleep apnea among obese patients with type 2 diabetes. Diabetes Care. 2009;32(6): 1017-1019.

23. Redline S, Sanders MH, Lind BK, et al. Methods for obtaining and analyzing unattended polysomnography data for a multicenter study. Sleep. 1998;21(7):759-767.

24. Kapur VK, Rapoport DM, Sanders MH, et al. Rates of sensor loss in unattended home polysomnography: the influence of age, gender, obesity, and sleep disordered breathing. Sleep. 2000;23(5):682-688.

25. Kushida CA, Littner MR, Morgenthaler T, et al. Practice parameters for the indications for polysomnography and related procedures: an update for 2005. Sleep. 2005;28(4):499-521.

26. [No authors listed]. Sleep-related breathing disorders in adults: recommendations for syndrome definitions and measurement techniques in clinical research. The Report of an American Academy of Sleep Medicine Task Force. Sleep. 1999;22(5):667-689.

27. Landis JR, Koch GG. An application of hierarchical kappa-type statistics in the assessment of majority agreement among multiple observers. Biometrics. 1977;33(2):363-374.

28. Sharma SK, Kumpawat S, Goel A, Banga A, Ramakrishnan L, Chaturvedi P. Obesity, and not obstructive sleep apnea, is responsible for metabolic abnormalities in a cohort with sleep-disordered breathing. Sleep Med. 2007;8(1):12-17.

29. Shahar E, Whitney CW, Redline S, et al. Sleep-disordered breathing and cardiovascular disease: cross-sectional results of the Sleep Heart Health Study. Am J Respir Crit Care Med. 2001;163(1):19-25.

30. Tsai YW, Kann NH, Tung TH, et al. Impact of subjective sleep quality on glycemic control in type 2 diabetes mellitus. Fam Pract. 2012;29(1):30-35.

31. Knutson KL, Ryden AM, Mander BA, Van Cauter E. Role of sleep duration and quality in the risk and severity of type 2 diabetes mellitus. Arch Intern Med. 2006;166(16):1768-1774.

32. Knutson KL, Van Cauter E, Zee P, Liu K, Lauderdale DS. Crosssectional associations between measures of sleep and markers of glucose metabolism among subjects with and without diabetes: the Coronary Artery Risk Development in Young Adults (CARDIA) Sleep Study. Diabetes Care. 2011;34(5):1171-1176.
33. Sharma SK, Agrawal S, Damodaran D, et al. CPAP for the metabolic syndrome in patients with obstructive sleep apnea. $N$ Engl J Med. 2011;365(24):2277-2286

34. Kohler M, Stoewhas AC, Ayers L, et al. Effects of continuous positive airway pressure therapy withdrawal in patients with obstructive sleep apnea: a randomized controlled trial. Am J Respir Crit Care Med. 2011; 184(10):1192-1199.

35. Michailidis V, Steiropoulos P, Nena E, Papanas N, Maltezos E, Bouros D. Continuous positive airway pressure treatment: effect on serum lipids in patients with obstructive sleep apnoea. Open Cardiovasc Med J. 2011;5:231-238.

36. Williams CJ, Hu FB, Patel SR, Mantzoros CS. Sleep duration and snoring in relation to biomarkers of cardiovascular disease risk among women with type 2 diabetes. Diabetes Care. 2007;30(5):1233-1240.

37. Newman AB, Nieto FJ, Guidry U, et al. Relation of sleep-disordered breathing to cardiovascular disease risk factors: the Sleep Heart Health Study. Am J Epidemiol. 2001;154(1):50-59.

38. Hall MH, Okun ML, Sowers M, et al. Sleep is associated with the metabolic syndrome in a multi-ethnic cohort of midlife women: the SWAN Sleep Study. Sleep. 2012;35(6):783-790.

39. Vgontzas AN, Zoumakis E, Bixler EO, et al. Selective effects of CPAP on sleep apnoea-associated manifestations. Eur J Clin Invest. 2008;38(8):585-595

40. Levendowski D, Steward D, Woodson BT, Olmstead R, Popovic D, Westbrook P. The impact of obstructive sleep apnea variability measured in-lab versus in-home on sample size calculations. Int Arch Med. 2009;2(1):2.

41. Quan SF, Griswold ME, Iber C, et al. Short-term variability of respiration and sleep during unattended nonlaboratory polysomnography - the Sleep Heart Health Study. [corrected]. Sleep. 2002;25(8):843-849.
Nature and Science of Sleep

\section{Publish your work in this journal}

Nature and Science of Sleep is an international, peer-reviewed, open access journal covering all aspects of sleep science and sleep medicine, including the neurophysiology and functions of sleep, the genetics of sleep, sleep and society, biological rhythms, dreaming, sleep disorders and therapy, and strategies to optimize healthy sleep. The journal welcomes

\section{Dovepress}

original research, clinical \& epidemiological studies, reviews \& evaluations, case reports and extended reports. The manuscript management system is completely online and includes a very quick and fair peerreview system, which is all easy to use. Visit http://www.dovepress.com/ testimonials.php to read real quotes from published authors. 